\title{
Renal Metastasis from Hurthle Cell Thyroid Carcinoma and Its Evaluation with Hybrid Imaging
}

\author{
Mehdi Djekidel,, ${ }^{1,2}$ Michael Gordon,, Rajal B. Shah,, ${ }^{3,4}$ Milton D. Gross, ${ }^{2,5}$ and Anca Avram²
}

Background: In general, thyroid carcinomas, when they behave aggressively, metastasize to lungs and occasionally to bone and brain. Metastases to other organs are distinctly unusual. Renal metastases have been reported in only 10 patients. Here we report, to the best of our knowledge, the first patient with Hurthle cell thyroid cancer and renal metastasis. The evaluation of this patient was enhanced by utilizing a variety of imaging techniques.

Summary: The patient was a 75-year-old man who had a history of a total thyroidectomy 9 years previously for a right thyroid lobe Hurthle cell carcinoma. He postoperatively received $150 \mathrm{mCi}(5550 \mathrm{MBq})$ of $131-\mathrm{I}$ therapy and was maintained on thyrotropin suppressive therapy with levothyroxine. He presented to us with a recent history of a progressively enlarging left neck mass. The serum thyroglobulin was elevated to $1183 \mathrm{ng} / \mathrm{mL}$. Multimodality imaging with fluorodeoxyglucose positron emission tomography-computed tomography, magnetic resonance imaging, and Somatostatin receptor scintigraphy with single photon emission computed tomography (SPECT)computed tomography revealed numerous foci in the skeleton and right kidney. Anatomic imaging characteristics favored a primary renal cell cancer with the additional evidence of renal vein invasion and thrombosis. Histology later revealed a metastatic renal Hurthle cell cancer with positive thyroglobulin stains. Several of the skeletal foci responded partially to cryoablative therapy. The patient refused noncurative Somatostatin analog therapy. He is alive and doing well clinically.

Conclusion: The management of thyroid cancers in high-risk groups, such as our patient, frequently requires expert management by the use of novel multimodality imaging and therapeutic techniques.

\section{Introduction}

$\mathbf{T}$

HYROID CANCER USUALLY HAS a favorable prognosis $(1,2)$.

The standard of care consists of total thyroidectomy with or without lymph node dissection, followed by postoperative radioiodine administration targeting the thyroid remnant and/or residual locoregional disease. Distant metastases are unusual at the initial presentation, and recurrence is significantly more frequent in locoregional lymph nodes and less common at distant sites (2). Kidney metastases are very rare, with only about 10 reported patients to date (3-5). Among these patients none had Hurthle cell thyroid carcinoma. Here we report a case of recurrent Hurthle cell carcinoma with renal metastasis.

\section{Patient}

The patient was a 75-year-old man who presented with recurrent disease 9 years after a total thyroidectomy performed in 1997 for excision of a right thyroid lobe mass; the surgically excised pathology specimen demonstrated Hurthle

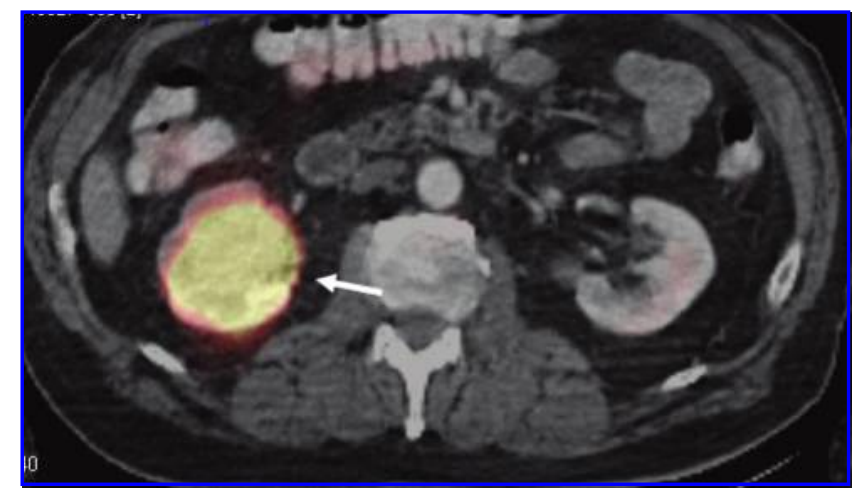

FIG. 1. Initial fluorodeoxyglucose positron emission tomography-computed tomography renal mass (white arrow). Hurthle cell metastatic focus. Color images available online at www.liebertonline.com/thy.

${ }^{1}$ Department of Radiology, Yale University School of Medicine, New Haven, Connecticut.

Departments of ${ }^{2}$ Radiology, ${ }^{3}$ Pathology, ${ }^{4}$ Urology, and ${ }^{5}$ Internal Medicine, University of Michigan, Ann Arbor, Michigan. 


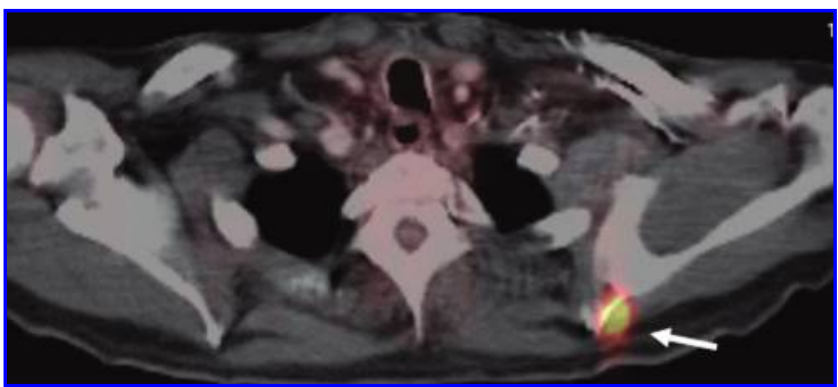

FIG. 2. Initial fluorodeoxyglucose positron emission tomography-computed tomography with a metabolically active left scapular lesion (white arrow). Color images available online at www.liebertonline.com/thy.

cell thyroid carcinoma. Postoperatively, the patient was treated with $150 \mathrm{mCi}(5550 \mathrm{MBq})$ 131-I and placed on levothyroxine (L-T4) suppressive therapy with L-T4 $0.15 \mathrm{mg}$ daily. Subsequently, the results of 131-I whole body scans in 1999,2001 , and 2003 were all negative and his thyroglobulin (Tg) levels remained undetectable. Other pertinent medical history was significant only for hypertension. The patient was retired, a nonsmoker, had no history of exposure to ionizing radiation, and had no family history of thyroid cancer or any other malignancy.
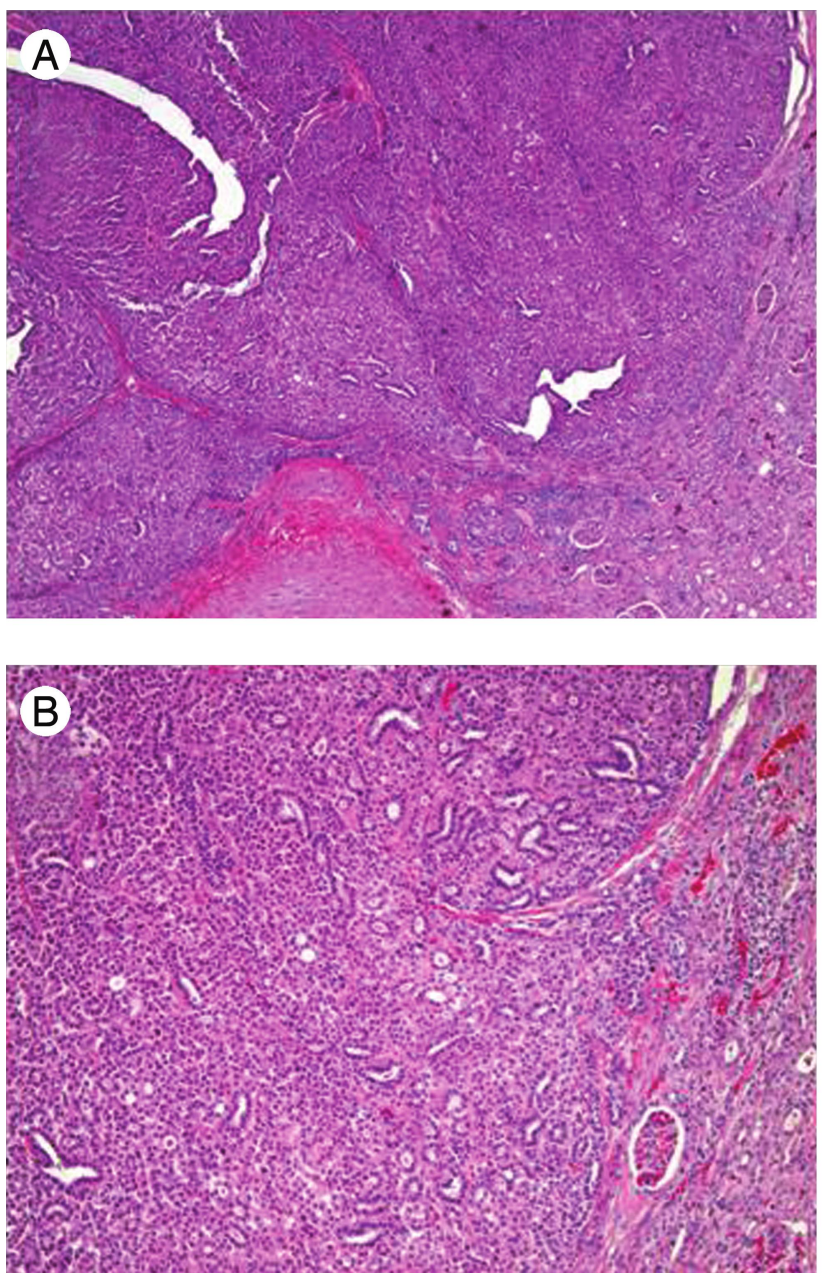

In early March 2006, clinical surveillance demonstrated gradual rise in Tg levels while under L-T4 suppression, prompting reevaluation with 131-I scan that revealed equivocal faint 131-I activity in the left neck, and administration of $150 \mathrm{mCi}(5550 \mathrm{MBq})$ 131-I for treatment. However, on subsequent clinical examination a left-sided neck mass became palpable, and fine-needle aspiration biopsy of this mass was suspicious for Hurthle cell neoplasm. The patient underwent excision of the left-sided neck mass on March 28, 2006, and the pathology specimen demonstrated recurrent Hurthle cell carcinoma infiltrating the skin and subcutaneous tissues. Despite surgical resection of the recurrent tumor in the neck, biochemical monitoring revealed progressively rising $\mathrm{Tg}$ levels to $985 \mathrm{ng} / \mathrm{mL}$ in June 2006 and to $1164 \mathrm{ng} / \mathrm{mL}$ in August 2006 while under L-T4 suppression therapy (thyrotropin [TSH]: $0.4 \mathrm{mIU} / \mathrm{L}$ ). Subsequent FDG PET-CT (fluorodeoxyglucose positron emission tomography-computed tomography) scan on September 11, 2006, revealed an intensely metabolically active right kidney mass suspicious for a renal cell carcinoma (Fig. 1). Additional metabolically active foci in the left scapula and the right acetabulum were consistent with skeletal metastatic disease (Fig. 2).

A magnetic resonance imaging revealed a large lobulated enhancing mass in the interpolar region and lower pole of the right kidney suspicious for a renal cell carcinoma invading the central renal sinus and suspicious for a tumor thrombus in

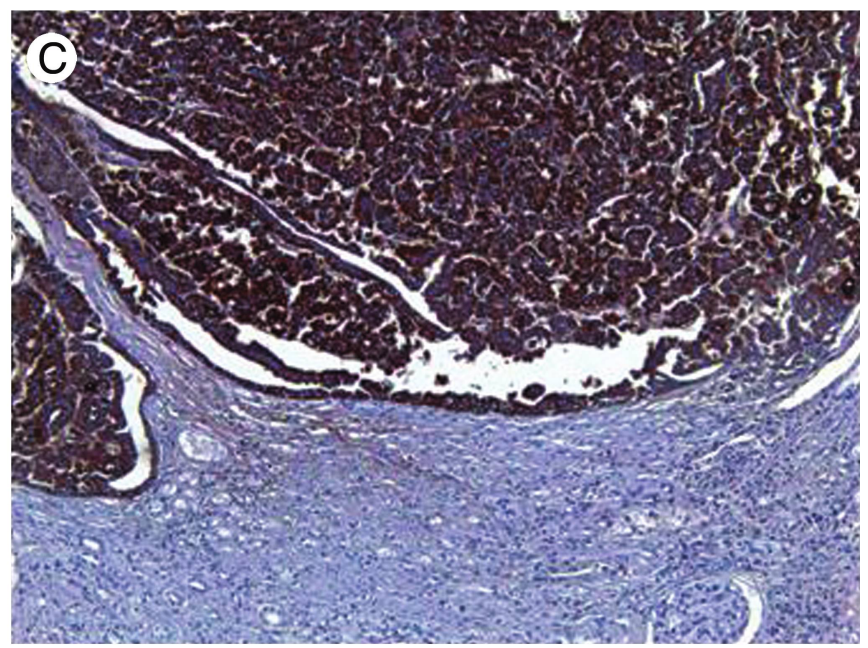

FIG. 3. Renal Hurthle cell cancer pathological specimen. (A) Low power view examination: relatively wellcircumscribed cellular tumor nodule composed of tubular structures and small cysts pushing the renal parenchyma seen in the right lower part of the image. Hematoxylin and eosin stain, $\times 40$. (B) Intermediate magnification tumor is composed of small tubules and acini lined by eosinophilic cytoplasm and uniform small nuclei. A portion of uninvolved renal parenchyma is seen in the right aspect of the image. Hematoxylin and eosin stain, $\times 100$. (C) Tumor cells demonstrate diffuse and intense reaction with thyroglobulin antibody, confirming the diagnosis of metastatic Hurthle cell carcinoma. The portion of uninvolved renal parenchyma lacks this expression. Immunoperoxidase stain $\times 100$. Color images available online at www.liebertonline.com/thy. 

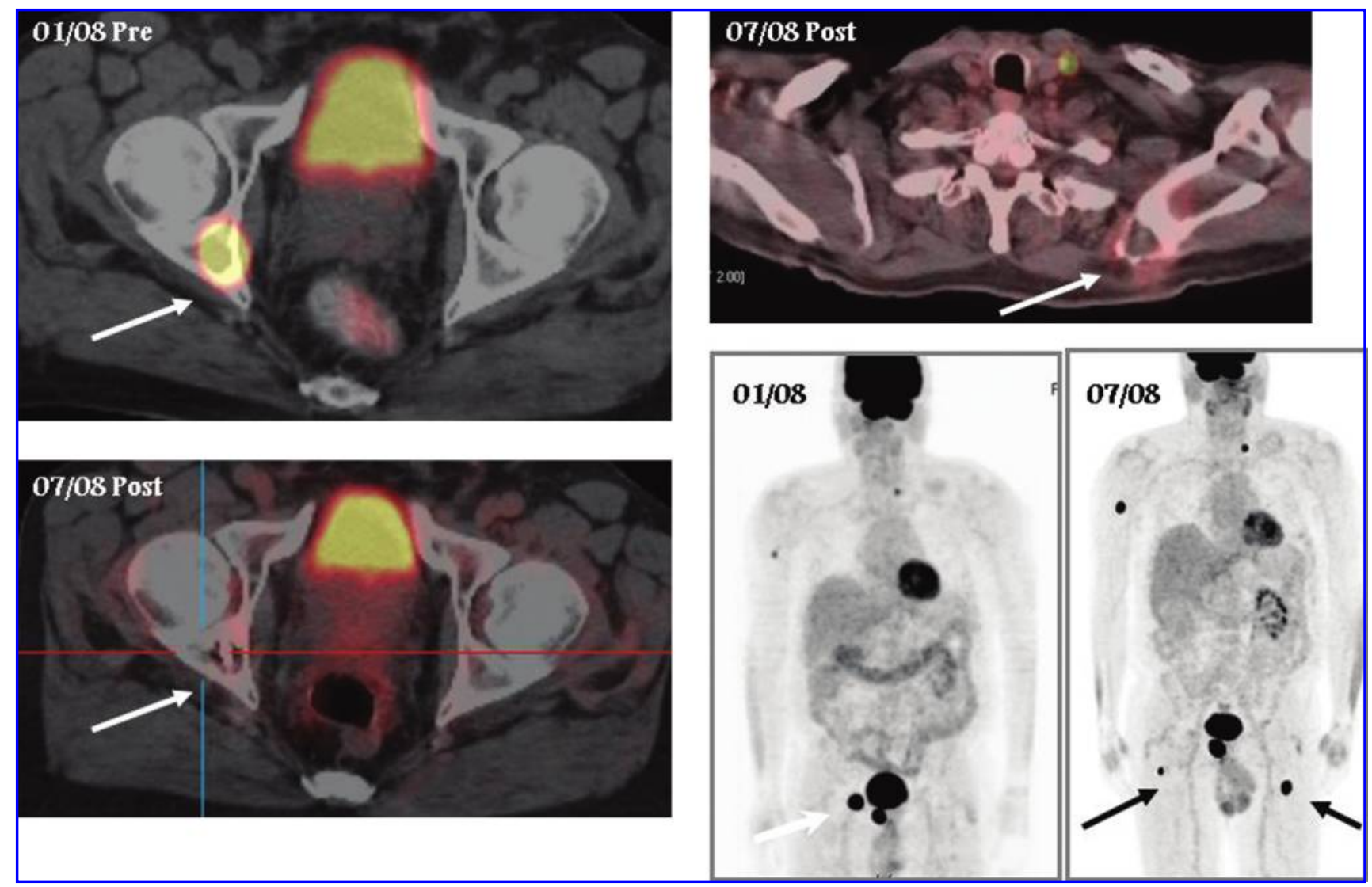

FIG. 4. Follow-up scan 6 months postcryoablation $\rightarrow$ Treatment. Response: right acetabulum and left scapula. 01/08 and 7/24/08 (white arrows) $\rightarrow$ new lesions (black arrows). Color images available online at www.liebertonline.com/thy.

the right renal vein but without extension into the inferior vena cava. Right radical nephrectomy and adrenalectomy on December 14, 2006, revealed a 5-cm tumor with a histological pattern consistent with Hurthle cell carcinoma and positive Tg staining (Fig. 3) involving the renal vein and invading the renal sinus fat. Vascular and ureteral margins were negative; the resected adrenal gland was negative for neoplastic involvement.

Immediately after surgery $\mathrm{Tg}$ levels decreased temporarily to $256 \mathrm{ng} / \mathrm{mL}$ (TSH: $0.09 \mathrm{mIU} / \mathrm{L}$ ) but rose again to $595 \mathrm{ng} / \mathrm{mL}$ (TSH: $0.14 \mathrm{mIU} / \mathrm{L}$ ), prompting another FDG PET scan on January 3, 2008, uncovering new metastatic lesions in the left supraclavicular fossa, right proximal humerus, and right pubic bone and interval worsening of abnormal FDG uptake in the right acetabulum. The left scapular lesion demonstrated a patchier uptake that extended into the adjacent soft tissues (Fig. 4).

In anticipation of possible Sandostatin long acting release (LAR) therapy, an Octreotide scan was performed, which revealed Somatostatin receptor expressing lesions in the left scapula, right pubic bone, right acetabulum, the right mid humerus, and the right posterior elements of C5 (Fig. 5). The patient underwent cryoablation of his scapular lesion and right acetabulum with some improvement; however, his disease continued to progress clinically with rising biomarkers. The Tg level was $974 \mathrm{ng} / \mathrm{mL}$ on July 22, 2008, when his TSH was $0.04 \mathrm{mIU} / \mathrm{L}$, and there was progression of disease on his follow-up FDG PET-CT scan (Fig. 4).
The patient refused Sandostatin LAR or biphosphonate therapy and opted for a holistic medicinal approach using dichloroacetate and vitamin C infusions. In August 2009, the patient presented with a pathologic fracture of the right humerus (Fig. 6). The patient declined surgical resection of the osseous metastatic lesion opting for a Sarmiento brace to stabilize the bone. In September 2009, the patient presented with difficulty ambulating and a focal sensory deficit in the left lower extremity, and a magnetic resonance imaging performed on September 28, 2009, demonstrated a new T3 vertebral lesion with encroachment upon the spinal cord (Fig. 6). He was treated with Decadron and conventional fractionated radiation therapy delivering a total of $30 \mathrm{~Gy}$ in daily $3 \mathrm{~Gy}$ fractions over the course of 2 weeks. Currently, he is clinically stable; however, his Tg levels continue to rise (most recent level on October 22, 2009: Tg $14113 \mathrm{ng} / \mathrm{mL}$; TSH $0.08 \mathrm{mU} / \mathrm{L}$ ) consistent with continued advancement of his metastatic disease.

\section{Discussion}

Kidney metastases are usually a rare occurrence and even less commonly of thyroid origin. Only 10 patients have been reported so far, 7 of follicular and 3 of papillary origin (3-5). Hurthle cell cancer is considered a variant of follicular cell thyroid cancer and represents 3\% of thyroid carcinomas; it is defined by the presence of more than $75 \%$ of oncocytic cells with an eosinophilic cytoplasm and a trabecular/follicular growth pattern. 


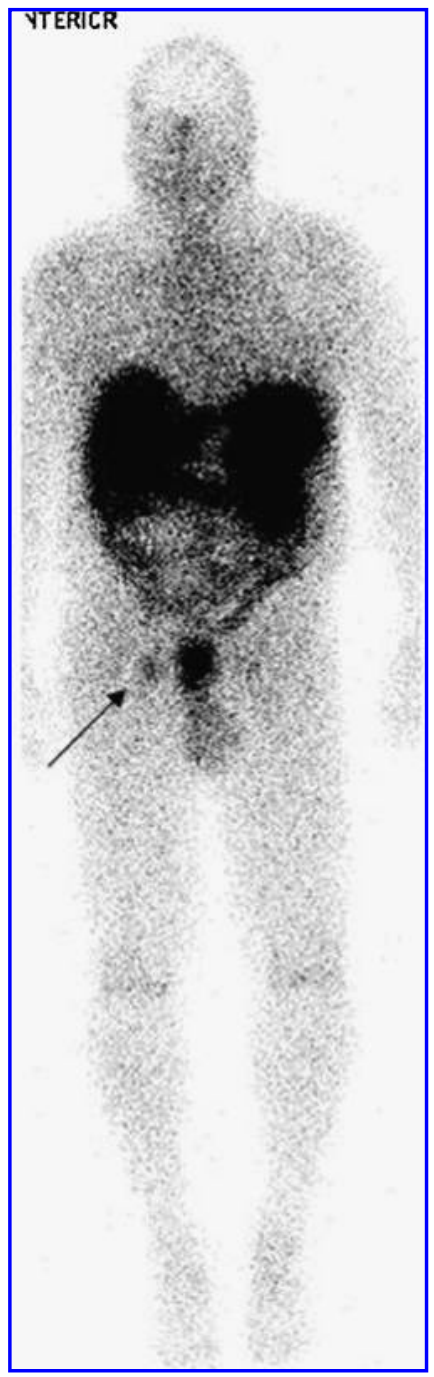

FIG. 5. Octreotide avid right acetabulum site (arrow).
With the advent of novel imaging techniques and therapy options, a more aggressive management approach and expert opinion involvement is necessary in the management of highrisk thyroid cancer patients. Our patient had several adverse prognostic indicators, including age, sex, the Hurthle cell histology, and distant aggressive metastatic disease involving the skeleton and a large renal metastasis occurring more than 5 years after initial diagnosis. His metastatic disease did not concentrate radioiodine, as evidenced by negative 131-I scans, and was highly metabolically active on fluorine-18-labeled FDG PET-CT scans.

Early-stage thyroid cancers have a benign course; however, in cases presenting with advanced (stage IV) disease where the tumor exhibits an aggressive biological behavior, expert multimodality imaging is essential for accurate assessment of the extent of disease, guiding treatment to halt progression and improve survival. Recently, fluorine-18-labeled FDG PET-CT has been shown to detect the aggressive transformation of thyroid cancers and uncover dedifferentiated disease characterized by a loss of capacity to concentrate iodine, the so-called noniodine avid thyroid cancer. FDG PET-CT may also occasionally be used to image these more aggressive iodine avid thyroid cancers $(6,7)$. Hormone withdrawal and recombinant human TSH stimulation have been used with variable success. Finally, somatostatin receptor scintigraphy may image various types of thyroid tumors (8), especially when $\mathrm{Tg}$ is above $10 \mathrm{ng} / \mathrm{mL}$. This appears to have the potential for therapy use with somatostatin analogs.

It is crucial to understand the natural course, genetics, and biology of these more aggressive tumors and develop adequate therapies. The current approach is aggressive tumor resection both locally and at distant sites, as it offers the best survival. If bony lesions are resected, a prosthesis may be inserted. The multitude and locations of the skeletal lesions in our patient limited this option.

Radiofrequency and cryoablation for various cancers has gained clinical interest recently. These procedures have been used to treat difficult metastases in liver, lung, renal, and

FIG. 6. (A) Right humeral pathological fracture (arrow). (B) T3 spinal mass (arrow).

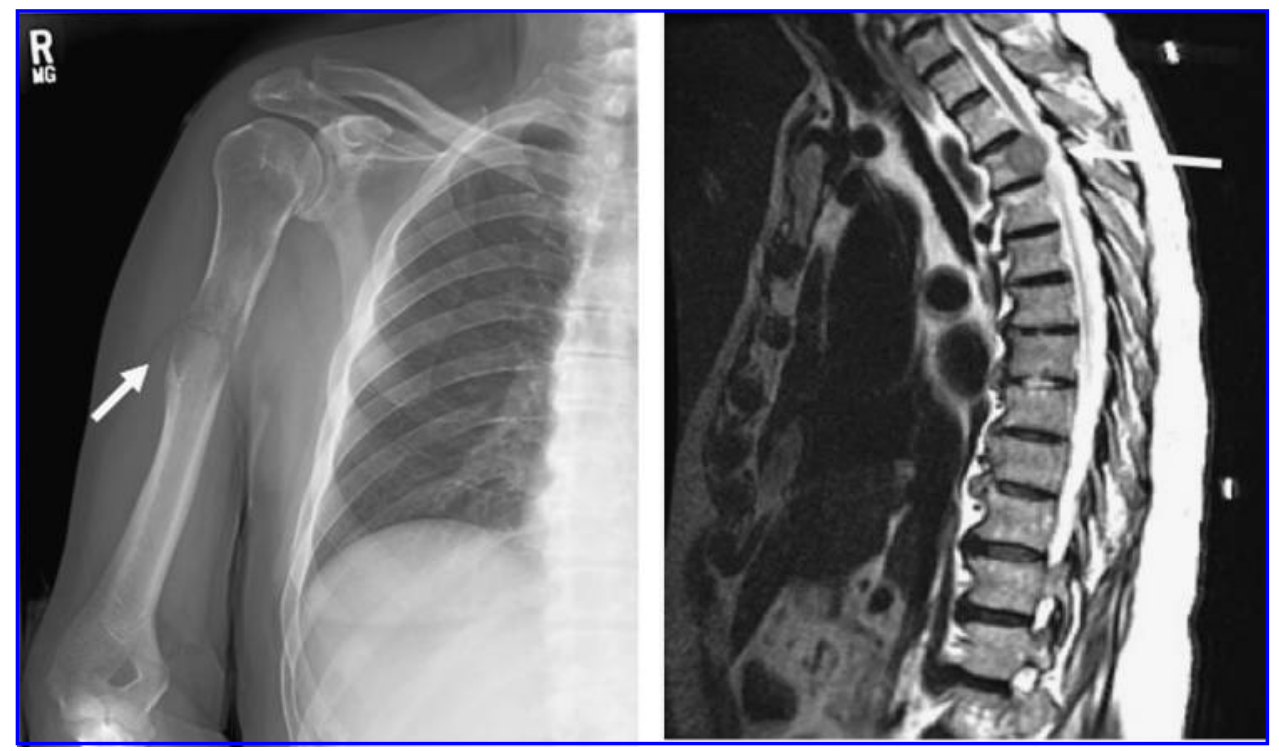


prostate cancers. They have rarely been used to treat thyroid metastasis, however $(9,10)$. They may alleviate pain and reduce tumor bulk, but survival data are not readily available. In our patient, cryoablation was used effectively with some relief of pain caused by multiple skeletal metastases.

In summary, the management of high-risk thyroid cancers can be enhanced by the use of novel multimodality imaging and therapeutic techniques.

\section{Disclosure Statement}

All authors have no relevant disclosures.

\section{References}

1. Shoup M, Stojadinovic A, Nissan A, Ghossein RA, Freedman S, Brennan MF, Shah JP, Shaha AR 2003 Prognostic indicators of outcomes in patients with distant metastases from differentiated thyroid carcinoma. J Am Coll Surg 197:191197.

2. Palme CE, Waseem Z, Raza SN, Eski S, Walfish P, Freeman JL 2004 Management and outcome of recurrent welldifferentiated thyroid carcinoma. Arch Otolaryngol Head Neck Surg 130:819-824.

3. Graham LD, Roe SM 1995 Metastatic papillary thyroid carcinoma presenting as a primary renal neoplasm. Am Surg 61:732-734.

4. Moudouni SM, En-Nia I, Rioux-Leclerq N, Manunta A, Guille F, Lobel B 2002 Follicular carcinoma of the thyroid metastasis to the kidney nine years after resection of the primary tumor. Ann Urol 36:36-37.
5. Abe K, Hasegawa T, Onodera S, Oishi Y, Suzuki M 2002 Renal metastasis of thyroid carcinoma. Int J Urol 9:656-658.

6. Lowe VJ, Mullan BP, Hay ID, McIver B, Kasperbauer JL 2003 F18-FDG PET of patients with Hurthle cell carcinoma. J Nucl Med 44:1402-1406.

7. Mirallié E, Guillan T, Bridji B, Resche I, Rousseau C, Ansquer C, Bodet-Milin C, Curtet C, Carnaille B, Murat A, Charbonnel B, Kraeber-Bodéré F 2007 Therapeutic impact of 18FDG-PET/CT in the management of iodine-negative recurrence of differentiated thyroid carcinoma. Surgery 142: 952-958.

8. Görges R, Kahaly G, Müller-Brand J, Mäcke H, Roser HW, Bockisch A 2001 Radionuclide labeled somatostatin analogues for diagnostic and therapeutic purposes in nonmedullary thyroid cancer. Thyroid 11:647-659.

9. Monchik JM, Donatini G, Iannuccilli J, Dupuy DE 2006 Radiofrequency ablation and percutaneous ethanol injection treatment for recurrent local and distant well-differentiated thyroid carcinoma. Ann Surg 244:296-304.

10. Gysin P, Gloor B 1979 Cryo- and photocoagulation for choroidal metastases of a thyroid carcinoma. Klin Monatsbl Augenheilkd 174:978-981.

Address correspondence to: Mehdi Djekidel, M.D. Department of Radiology Yale University School of Medicine P.O. Box 208042

New Haven, CT 06520-8042

E-mail: mehdi.djekidel@yale.edu. 

This article has been cited by:

1. Mehdi Djekidel , Anca M. Avram . 2010. Response to Malhotra et al.Response to Malhotra et al.. Thyroid 20:11, 1321-1322. [Citation] [Full Text] [PDF] [PDF Plus]

2. Gaurav Malhotra, Ramesh Asopa , Mysore Govinda Ramakrishna Rajan . 2010. Renal Metastases from Thyroid CarcinomaRenal Metastases from Thyroid Carcinoma. Thyroid 20:11, 1321-1321. [Citation] [Full Text] [PDF] [PDF Plus] 